\title{
tic\&société
}

Vol. 12, $\mathrm{N}^{\circ} 2$ | 2ème semestre 2018

Numérique et situations de handicap : les enjeux de l'accessibilité

\section{Sourds et malentendants comme publics de la musique. Le statut ambigu des technologies numériques dans une démarche d'accessibilité}

Mélanie Hénault-Tessier, Thibault Christophe et Nathalie NEGREL

\section{OpenEdition}

Édition électronique

URL : http://journals.openedition.org/ticetsociete/2877

DOI : 10.4000/ticetsociete. 2877

Éditeur

Association ARTIC

Édition imprimée

Pagination : 75-102

Référence électronique

Mélanie Hénault-Tessier, Thibault Christophe et Nathalie NEGREL, « Sourds et malentendants comme publics de la musique. Le statut ambigu des technologies numériques dans une démarche d'accessibilité », tic\&société [En ligne], Vol. 12, № 2 I 2ème semestre 2018, mis en ligne le 10 décembre 2018, consulté le 30 avril 2019. URL : http://journals.openedition.org/ticetsociete/2877 ; DOI :

$10.4000 /$ ticetsociete. 2877 
tic\&société - 12(2), 2018

\title{
Sourds et malentendants comme publics de la musique. Le statut ambigu des technologies numériques dans une démarche d'accessibilité
}

\begin{abstract}
Mélanie Hénault-Tessier
Mélanie Henault-Tessier est chercheuse associée au laboratoire Laboratoire d'études et de recherches appliquées en sciences sociales (LERASS) de l'Université Toulouse Jean-Jaurès. Ses travaux de recherche à la croisée de la sociologie et de l'information-communication portent sur les processus conjoints de transformations des pratiques, des espaces et des organisations engendrés par la prise en compte des spécificités sociolinguistiques des personnes sourdes au sein de différents mondes sociaux, notamment le travail et la culture.

projetmuvu@gmail.com
\end{abstract}

\section{Thibault Christophe}

Thibault Christophe est chercheur associé au Laboratoire d'études et de recherches appliquées en sciences sociales (LERASS) de I'Université Toulouse Jean-Jaurès. Après avoir mené une thèse en information-communication sur les pratiques d'écoute musicales des adolescentes et adolescents en régime numérique, il poursuit désormais des recherches sur la dialogie "musique et minorité " abordée à travers deux thématiques: les objets vidéo queer faisant appel aux musiques populaires et les pratiques d'écoute musicale des personnes sourdes. tibo.christophe@univ-tlse2.fr 
Sourds et malentendants comme publics de la musique. Le statut ambigu des technologies numériques dans une démarche d'accessibilité

\section{Nathalie NEGREL}

Nathalie Negrel est chercheuse associée au Laboratoire d'études et de recherches appliquées en sciences sociales (LERASS) de I'Université Toulouse Jean-Jaurès et enseignante en expressioncommunication à l'Institut national des sciences appliquées Toulouse. Elle est également directrice du Centre Gaston Berger, chargé de décliner la notion de diversité au sein de l'établissement. Ses travaux de recherche ont porté sur les représentations télévisuelles transnationales des migrations subsahariennes et s'actualisent aujourd'hui autour de la problématique de l'accessibilité culturelle.

nathalie.negrel@insa-toulouse.fr 
Mélanie HÉNAULT-TESSIER, Thibault CHRISTOPHE et Nathalie NEGREL

\section{Sourds et malentendants comme publics de la musique. Le statut ambigu des technologies numériques dans une démarche d'accessibilité}

Résumé : La notion d'accessibilité culturelle soulève des questions qui s'articulent aujourd'hui aux problématiques liées aux technologies numériques. Dans le champ du handicap, l'accessibilité culturelle par le numérique souffre toutefois du peu de recherches, notamment de recherches empiriques. Les besoins, les pratiques et l'avis des personnes concernées par les processus d'accessibilité restent donc largement méconnus, ce qui maintient une certaine ambiguïté sur le rôle et le statut des technologies dans le cours de ces processus. En prenant comme point d'entrée les expériences musicales des personnes sourdes et malentendantes, observées lors d'un festival hip-hop, nous analysons en quoi le numérique participe de ces expériences musicales singulières et comment celles-ci interrogent en retour la notion d'accessibilité. Nous verrons ainsi que les enjeux spécifiques des technologies numériques résident dans le fait qu'elles contribuent à la fois à définir les processus d'accessibilité et leurs utilisateurs.

Mots-clés: musique, surdité, technologies numériques, accessibilité, appropriabilité.

Abstract: The notion of cultural accessibility raises questions that are linked to issues related to digital technologies. However, in the field of disability, cultural accessibility through digital technology suffers from a lack of research, particularly empirical research. As a result, the needs, practices and opinions of those concerned by accessibility processes remain largely unknown, and the role and status of technologies in these processes remains ambiguous. Taking the musical experiences of deaf and hard of hearing audiences during a hip-hop festival, as an entry point, we question how digital technology participates in their singular musical experiences. We observe that these experiences challenge the notion of accessibility and that digital technologies' contribution to 
Sourds et malentendants comme publics de la musique. Le statut ambigu des technologies numériques dans une démarche d'accessibilité

both defining accessibility processes and their users raises specific issues.

Keywords: music, deafness, digital technologies, accessibility, appropriability.

Resumen: El concepto de accesibilidad cultural plantea preguntas que están relacionadas con problemáticas vinculadas a las tecnologías digitales. No obstante, en el campo de la discapacidad, existen escasas investigaciones sobre la accesibilidad cultural a través de lo digital, y en especial las investigaciones empíricas. Las necesidades, las prácticas y las opiniones de las personas afectadas por los procesos de accesibilidad continúan siendo en gran parte desconocidas; lo que muestra una cierta ambigüedad en torno al rol y al estado de las tecnologías en dichos procesos. Partiendo de las experiencias musicales, observadas durante un festival de hip-hop, de las personas con dificultades auditivas o sordas, el artículo analiza cómo influye la tecnología digital en estas experiencias musicales singulares y cómo éstas experiencias plantean el problema de la accesibilidad; y concluye que los desafíos específicos de las tecnologías digitales se basan en el hecho de que contribuyen a definir no sólo los procesos de accesibilidad sino también sus usuarios.

Palabras clave: música, sordera, tecnologías digitales, accesibilidad, apropiación. 
Mélanie HÉNAULT-TESSIER, Thibault CHRISTOPHE et Nathalie NEGREL

\section{Introduction}

\section{L'accès à la culture pour les personnes handicapées : un droit peu encadré}

En France, ces dernières décennies ont donné lieu au développement et à la formalisation « d'une accessibilité construite et perçue comme un droit par les personnes handicapées » (Larrouy, 2007, p. 29). D'abord appréhendé du point de vue du cadre bâti et des transports, le thème de l'accessibilité a ensuite "été amplifié comme thème sociopolitique de défenses des personnes en situation de handicap " (Plaisance, 2015, p. 87) avant d'être finalement mis au cœur du principe général d'égalité des droits et des chances ${ }^{1}$.

Les textes législatifs français de référence dans le domaine du handicap (la loi de 1975 et la loi de février 2005) demeurent toutefois timides au sujet de la culture (Duranton et GonthierMaurin, 2017 ; Plaisance, 2015). Ils ne comportent en effet aucune disposition claire destinée à en faciliter l'accès. Ils réaffirment cependant le principe d'égalité des droits et des chances qui, par extension, inclut l'accès à la culture déjà inscrit au Préambule de la Constitution ${ }^{2}$. Ce droit d'accès aux lieux culturels, aux œuvres et à une pratique artistique pour les personnes handicapées est par ailleurs reconnu plus explicitement par trois textes normatifs européens et internationaux ${ }^{3}$.

\footnotetext{
${ }^{1}$ À titre d'exemple, le Programme d'action mondial concernant les personnes handicapées adopté par les Nations Unies en 1982 associe égalisation des chances et accessibilité en faisant de celle-ci le résultat du processus d'égalisation : "Le terme "égalisation des chances" désigne le processus par lequel le cadre général de la société - environnement matériel et culturel, logement et transports, services sociaux et services de santé, enseignement et emplois, et aussi la vie culturelle et sociale, y compris les installations sportives et les équipements de loisirs - est rendu accessible à tous. » (p. 6.) ${ }^{2}$ Le Préambule de la Constitution de 1946 confirme que « [l]a Nation garantit l'égal accès de l'enfant et de l'adulte à l'instruction, à la formation professionnelle et à la culture. » ${ }^{3}$ La Résolution du Conseil de I'Union européenne du 6 mai 2003, la Résolution de l'Organisation des Nations Unies (ONU) du 29 décembre 1993 et la Convention de l'ONU relative aux personnes handicapées du 13 décembre 2006.
} 
Sourds et malentendants comme publics de la musique. Le statut ambigu des technologies numériques dans une démarche d'accessibilité

La traduction en termes pratiques de ce droit est ainsi peu encadrée. Le premier guide institutionnel portant sur l'accessibilité culturelle n'a, par exemple, été publié qu'en 2007 par le ministère de la Culture ${ }^{4}$. L'accessibilité culturelle renvoie donc encore aujourd'hui à des lieux, des actions, des acteurs et des dispositifs variés. Souffrant d'une double minoration puisqu'elle ne concerne qu'une faible proportion de la population et qu'elle ne semble pas prioritaire par rapport à d'autres besoins, en particulier l'éducation et l'emploi (Dubois et Ebersold, 2013; Duranton et GonthierMaurin, 2017), elle demeure généralement négligée et pâtit d'un déficit de moyens humains et financiers. Celle-ci dépend alors " des aléas d'initiatives individuelles clairsemées, du bénévolat, d'appels à projets » (Fertier, 2013, p. 38). Cette situation contribue de la diversité, mais aussi de l'inégalité qualitative et territoriale des propositions en plus d'exclure les personnes handicapées " de l'offre culturelle relevant des services publics (ou en délégation de service public), du droit commun » (p. 38).

Le rapport d'information Culture et handicap remis au Sénat en 2017 précise cependant que « la question de l'accès à la culture des personnes en situation de handicap reste principalement abordée sous le prisme de l'accessibilité physique " (Duranton et Gonthier-Maurin, 2017, p. 10) malgré la diversité des initiatives. Cette perspective peut être expliquée par la genèse de la notion d'accessibilité, étroitement liée à une redéfinition environnementale du handicap (Albrecht, Ravaud et Stiker, 2001), mais aussi par le fait que les établissements culturels sont soumis aux obligations légales d'accès, de circulation et d'accueil concernant les établissements recevant du public (ERP) ${ }^{5}$. Or

\footnotetext{
${ }^{4}$ Ministère de la Culture et de la Communication, France (2007). Culture et handicap. Guide pratique de l'accessibilité. Repéré à https://www.monatourisme.fr/wpcontent/uploads/2010/12/CL_01-6____MIN_07-02Guide pratique d acces a la culture.pdf

${ }^{5}$ L'article L. 111-7-3. de la loi du 11 février 2005 pour l'égalité des droits et des chances, la participation et la citoyenneté des personnes handicapées stipule que « [l]es établissements existants recevant du public doivent être tels que toute personne handicapée puisse y accéder, y circuler et y recevoir les informations qui y sont diffusées, dans les parties ouvertes au public. L'information destinée au public doit être diffusée par des moyens adaptés aux différents handicaps ». La suite de l'article L. 111-7-3 apporte les précisions suivantes : « [...] Pour faciliter l'accessibilité, il peut être fait recours aux nouvelles technologies de la communication et à une signalétique adaptée ».
} 
Mélanie HÉNAULT-TESSIER, Thibault CHRISTOPHE et Nathalie NEGREL

l'accessibilité culturelle est aussi « une question de scénographie, de médiation humaine et de mise en œuvre de dispositifs techniques " (Ministère de la Santé et des Solidarités, Ministère délégué à la Sécurité sociale, aux Personnes âgées, aux Personnes handicapées et à la Famille, 2006, p. 9).

\section{Le numérique, nouvel outil de la pleine participation culturelle?}

Si la médiation humaine est depuis longtemps l'une des clés des politiques de démocratisation culturelle et l'une des modalités de l'exercice de la démocratie culturelle (Caune, 2017), les technologies sont désormais amplement mobilisées dans les processus de mise en accessibilité des œuvres et des contenus culturels.

Dans le champ du handicap, un corpus grandissant de travaux de recherche montre "que les situations de handicap se construisent généralement dans un environnement pauvre en moyens d'information et de communication adaptés " (Darras et Valente, 2013, p. 8). Le comité des droits économiques, sociaux et culturels de l'ONU insiste quant à lui sur le fait que l'accessibilité « comprend aussi le droit de chacun de rechercher, de recevoir et de partager des informations sur toutes les manifestations culturelles dans la langue de son choix " (Comité des droits économiques, sociaux et culturels, 2009, p. 5). Ces analyses et ces observations donnent sens aux démarches d'accessibilité par le numérique. Les technologies apparaissent ainsi comme une solution pour produire et diffuser une information adaptée aux spécificités individuelles. Au-delà de ces problématiques infocommunicationnelles, les technologies numériques sont également mobilisées comme " outils permettant l'aménagement de l'espace social, un moyen de gestion de l'altérité concrétisant les volontés et discours de l'État " (Hénault-Tessier et Dalle-Nazébi, 2013, p. 261). La numérisation des collections encourage par exemple la mise en contact entre des œuvres et des publics jusque-là exclus de certains lieux culturels, bien qu'il soit reproché à ce mode de mise en rapport d'isoler le visiteur. De nombreux lieux culturels, et plus particulièrement les musées, se dotent par ailleurs d'outils 
Sourds et malentendants comme publics de la musique. Le statut ambigu des technologies numériques dans une démarche d'accessibilité

numériques afin d'améliorer l'accueil et les expériences de visite des personnes handicapées ${ }^{6}$.

En permettant la création de nouvelles relations entre les contenus culturels et les publics, les technologies semblent inverser la dynamique de "qui s'adapte à qui ? " (Darras et Valente, 2013, p. 7). Si elles bénéficient d'un a priori favorable, le rôle et le statut de ces technologies dans les processus de mise en accessibilité demeurent cependant méconnus. Les enquêtes s'intéressant aux rapports qu'entretiennent les personnes concernées avec les œuvres et les productions culturelles rendues accessibles sont en effet encore peu fréquentes (Plaisance, 2015): l'attention est davantage portée sur l'évolution des politiques publiques et les processus institutionnels de mise en accessibilité que sur les expériences culturelles situées. Afin d'apporter des éléments de réponse sur la manière dont ces technologies participent du passage de l'énoncé du droit à la pleine participation culturelle à sa mise en œuvre pratique, nous observerons, dans cet article, par quels processus et à quelles conditions elles deviennent, en situation, des outils soutenant l'accès aux œuvres et aux évènements culturels, mais aussi leur appropriation.

Plus précisément, en prenant comme point d'entrée les expériences musicales des publics sourds et malentendants ${ }^{7}$, observées lors du festival Hip OPsession ${ }^{8}$, nous explorerons en quoi les technologies numériques participent de leurs expériences musicales singulières. En conservant notre attention sur la matérialité des pratiques d'écoute, nous montrerons d'abord en quoi les technologies contribuent à l'amplification et à la

\footnotetext{
${ }^{6}$ À titre d'exemple : le jeu Muséo expérimenté au Quai Branly permet aux enfants sourds d'explorer les œuvres à partir d'interfaces numériques tactiles (Houriez, Houriez, Kounakou et Leleu-Merviel, 2013) et le « compagnon de voyage » de la Cité du vin à Bordeaux, grâce à l'utilisation d'un boîtier numérique, offre une version sous-titrée des informations sonores ainsi qu'une version en français simplifié.

${ }^{7}$ Cette recherche sur « les expériences musicales sourdes et leurs dispositifs », menée du $1^{\text {er }}$ janvier au 31 décembre 2017 au sein du laboratoire LERASS de l'Université de Toulouse 3, a bénéficié d'une allocation postdoctorale du LabEx SMS. Elle a toutefois été menée collégialement grâce à un soutien financier supplémentaire par l'équipe de recherche Médiapolis-Grecom.

${ }^{8}$ Voir : http://www.hipopsession.com/.
} 
Mélanie HÉNAULT-TESSIER, Thibault CHRISTOPHE et Nathalie NEGREL

multiplication des «prises » musicales (Bessy et Chateaureynaud, 1995), mais que leur pertinence dépend avant tout de leur capacité à s'agencer et à être agencées aux situations, aux autres dispositifs, aux capacités et aux dispositions individuelles. Nous interrogerons ensuite plus particulièrement les technologies d'amplification sensorielle et soulignerons qu'elles peuvent paradoxalement être appréhendées comme prothèse ou comme mode de reformulation et de légitimation des rapports à la musique. Nous reviendrons finalement sur la notion d'accessibilité, remise en question et négociée par ces expériences musicales, ce qui nous permettra d'identifier quelques enjeux spécifiques de l'accessibilité culturelle par le numérique.

Par son attention au rôle des objets, dont les technologies numériques, dans la construction d'une relation au sensible et par les méthodes qui permettent de mettre l'engagement des usagers au centre de l'analyse, cette étude s'inscrit à la suite des travaux en sociologie du goût et de l'écoute musicale (Hennion, 1993, 2004 ; Hennion, Maisonneuve et Gomart, 2000 ; Maisonneuve, 2012). En faisant dialoguer ce corpus avec la sociologie du handicap et les Deaf Studies, cette étude s'attache par ailleurs à distinguer la notion d'accessibilité de la notion de médiation telle qu'elle est aujourd'hui appréhendée par les SIC (Caune, 2017 ; Davallon, 2003)

\section{La musique au-delà de l'oreille. Rendre un festival de musique accessible aux sourds et malentendants}

Le festival Hip OPsession se déroule annuellement à Nantes depuis 2005. II est consacré, comme son nom le suggère, à la diffusion de la culture hip-hop et a fait de l'accueil des sourds et malentendants l'une de ses priorités depuis plusieurs années ${ }^{9}$. Contrairement aux idées reçues, la musique fait partie de leur quotidien, qu'ils aient ou non un goût pour celle-ci, qu'ils perçoivent ou non ses éléments sonores. L'absence de rapports sonores au monde n'induit donc aucunement l'absence de rapports au monde sonore comme le révèle le récit d'une première rencontre avec le

${ }^{9}$ Les premières initiatives en ce sens remontent à 2007. 
Sourds et malentendants comme publics de la musique.

Le statut ambigu des technologies numériques dans une démarche d'accessibilité

spectacle qu'offre la musique classique jouée par un orchestre :

Le mouvement du percussionniste qui tape sa cymbale... je le regarde, il a l'air bête à taper comme ça. Et, l'autre, avec son triangle, ting-ting-ting - ting, ting, ting. Je les regarde et j'essaie d'imaginer le mouvement de la musique et le rôle de chacun des instruments. Le triangle produit une musique douce. Quand arrivent les violons, parfois il y en a quatre en même temps, le rythme est saccadé, puis effréné. Ces modulations, ça, j'aime ça! (Marc, 48 ans, sourd signant)

Les rapports que les sourds et malentendants entretiennent à la musique défient ainsi les partis pris audiocentrés sur ce que signifie écouter ${ }^{10}$.

La culture hip-hop se joue également de ces partis pris puisque le fait sonore y " entretient de multiples relations avec le visuel " (Boidy, Meursault et Pailler, 2015, p. 8), notamment à travers la danse, mais également des codes gestuels (Kauffman, 2004), le mixage de disques vinyle, des normes vestimentaires et des pratiques graphiques, dont la plus emblématique est le graffiti. D'ores et déjà, il apparaît que, pour «rendre accessible la musique " aux sourds et malentendants, il est possible de déplier le fait sonore afin de révéler les multiples relations qu'il entretient avec le visuel (nous aborderons plus loin la question des vibrations), ce qui encourage par ailleurs une mise en contact avec les esthétiques et les pratiques associées à la musique.

Analyser l'accessibilité musicale en pratiques suppose d'appréhender la musique non pas comme une œuvre en soi, mais comme une expérience qui s'appuie à la fois sur un rapport auditif et cognitif au fait sonore et sur une culture matérielle qui participe de la multiplication des modalités relationnelles ${ }^{11}$. Le disque vinyle

\footnotetext{
${ }^{10}$ La Langue des signes française (LSF) est à ce sujet très explicite puisque le signe « écouter » se réalise au niveau de l'œil.

${ }^{11}$ Dans le cadre de cet article, nous appréhendons la musique dans son acception complexe mais cependant restrictive. En effet, si la musique est « produit de dispositifs, d'activités multiples et multiformes qui se procurent mutuellement appui pour réaliser, faire advenir les choses : un "joyeux fouillis" d'énonciations, de personnages, de lieux, de moments, de bâtiments, d'équipements, d'appareils ou de machines, de matériaux, de papier, de controverses... » (Cheyronnaud, 2009, p. 8), elle conserve son acception classique de " son humainement organisé » (Blacking, cité dans Cheyronnaud, 2009,
} 


\section{Mélanie HÉNAULT-TESSIER, Thibault CHRISTOPHE et Nathalie NEGREL}

constitue un bon exemple de cette culture matérielle: leur son caractéristique, " la taille et l'esthétique des pochettes, ainsi que les gestes requis pour le jouer" sont autant de facteurs qui participent de l'expérience musicale (Nowak, 2013, p. 6). Au-delà de l'oreille, le fait sonore s'actualise à travers des rapports corporels, visuels, ou encore visuo-scriptuels ${ }^{12}$. L'analyse de l'écoute musicale en ce qui a trait à l'expérience induit également de l'appréhender comme une "épreuve qui passe entre producteur, auditeur et musique, et qui s'en trouve elle-même modifiée à travers des processus de façonnement et de structuration mutuels entre ces trois pôles » (Pecqueux et Roueff, 2006 , p. 16). Selon cette perspective, la musique "reste fondamentalement singulière en tant qu'elle nécessite d'être sentie, vécue, incorporée, pour devenir musique " (Brétéché, 2015 , p. 16). Les usagers sont ainsi définis comme acteurs de leurs propres goûts et plaisirs.

\subsection{Une enquête à l'écoute de l'écoute des sourds}

Interroger le rôle et la place que tiennent les technologies numériques au sein des expériences musicales des sourds et malentendants nécessite donc de se mettre à l'écoute de leur écoute afin d'en saisir les conditions pratiques et la diversité.

Ainsi, cette analyse s'appuie sur la parole des sourds et malentendants recueillie lors de 16 entretiens filmés, réalisés directement en langue des signes française (LSF) ou en présence d'un interprète français/LSF. La démarche empirique au cœur de cet article a parallèlement été développée afin de contourner l'effet déclaratif des entretiens. Des observations ont été réalisées lors d'évènements musicaux accessibles : au festival Hip OPsession,

p. 7). Cette définition est cependant remise en question par certains sourds créant des musiques visuelles, désignées par le néologisme "vusique », à travers l'articulation organisée d'images et de langue des signes reprenant les conventions de rythmicité, de répétition et d'intensité de la musique.

12 Les rapports visuo-scriptuels se réalisent à travers une relation à la fois visuelle et textuelle à la musique, c'est par exemple le cas lors de la lecture des paroles imprimées sur la pochette d'un disque vinyle ou lors de l'activation des sous-titres lors du visionnage d'un vidéoclip. 
Sourds et malentendants comme publics de la musique.

Le statut ambigu des technologies numériques dans une démarche d'accessibilité

au festival Clin d'œil à Reims ${ }^{13}$ et lors de deux concerts du groupe toulousain des Brassen's not dead. D'autres observations ont aussi été effectuées lors de moments de sociabilité entre personnes sourdes : soirées au bar, anniversaires et mariage. En plus de permettre la constitution d'un corpus d'images vidéo, ces observations ont été l'occasion de réaliser de courts entretiens en LSF $^{14}$ lors desquels les personnes sourdes et malentendantes étaient invitées à qualifier de manière spontanée leurs usages et leurs non-usages des solutions d'accessibilité proposées, à décrire leurs pratiques d'écoute musicale ordinaires et à détailler leurs motivations à participer à l'évènement.

Ces observations in situ sont apparues essentielles à l'analyse. S'il est intéressant d'apprendre que "les vibrations donnent des émotions ", cela est encore autre chose de voir un festivalier choisir avec soin la bonne distance à laquelle se tenir par rapport aux enceintes et à la scène. L'analyse a donc été enrichie par la mise en dialogue des données discursives et des observations. Par ailleurs, le caractère diffus du numérique le rend difficilement saisissable à travers les entretiens, qui tendent à ne révéler que ses éléments les plus représentatifs. II apparaît souvent, comme c'est le cas au festival Hip OPsession, à travers des hybridations analogico-numériques et en relation avec des objets matériels d'usage courant, des lieux et des pratiques. II se repère donc plus aisément lorsqu'il est abordé de manière empirique, et, surtout, sa pertinence, dans un projet d'accessibilité, ne peut être comprise qu'en situation.

\subsection{Des articulations qui font sens}

La mise en accessibilité de la programmation du festival Hip OPsession repose sur l'articulation de dispositifs hybrides d'accessibilité linguistique et d'amplification sensorielle à une pluralité de médiations humaines.

Dès l'entrée, les visiteurs sourds signants sont accueillis en LSF

\footnotetext{
${ }^{13}$ Le festival Clin d'œil est un festival d'art en langue des signes organisé par des sourd et dont les soirées sont rythmées par d'importantes Deaf Party, où la musique occupe une place importante.

${ }_{14}$ Quinze entretiens d'une quinzaine de minutes en moyenne ont été réalisés.
} 


\section{Mélanie HÉNAULT-TESSIER, Thibault CHRISTOPHE et Nathalie NEGREL}

par une médiatrice sourde et une bénévole signante. L'animation par un maître de cérémonie qui accompagne les battles affrontements d'équipes de danseurs devant un jury à l'issue desquels une équipe est déclarée gagnante - est quant à elle traduite en LSF par des interprètes qui, depuis un petit studio dissimulé dans les coulisses, traduisent devant une caméra vidéo les noms des danseurs, les styles de danse, les commentaires des jurés, etc. Loin d'être une simple interprétation rendant accessible le contenu parlé aux spectateurs sourds et malentendants, les interprètes proposent une restitution visuelle des expressions linguistiques et des intonations propre à la culture hip-hop. Cette interprétation est diffusée en temps réel sur deux écrans de taille importante qui jouxtent deux écrans géants, situés au fond et à droite de la scène, sur lesquels sont rediffusées les battles et les messages publicitaires des différents partenaires. Un troisième écran de plus petite taille est situé un peu à l'écart, dans la zone de détente où les rediffusions peuvent être regardées assis sur une chaise longue. Des sacs à dos ${ }^{15}$ et du mobilier vibrant sont aussi mis à la disposition des spectateurs. L'utilisation du numérique, qui s'articule à des pratiques professionnelles et linguistiques reconnues, prend notamment la forme d'une captation et d'une diffusion vidéo, ce qui constitue une utilisation de technologies d'usages courants à des fins d'accessibilité. En cela, il soutient le processus de multiplication des prises visuelles qui permet une mise en accessibilité linguistique du contenu, mais aussi une médiation, par la LSF, des manières de dire et, donc, une meilleure compréhension de la prosodie du hip-hop et le partage des émotions associées.

La pertinence de ce dispositif hybride est relationnelle. La présence des deux écrans géants servant tour à tour à la diffusion des battles et à la promotion du sac à dos vibrant, les gestes bien visibles du DJ juché sur son estrade, les jeux de lumière, l'espace circulaire créé par les danseurs au sein duquel les figures

\footnotetext{
${ }^{15}$ Des sacs à dos Subpack sont prêtés aux spectateurs qui le demandent. Ils retransmettent de manière vibratoire les basses fréquences de n'importe quelle source sonore, issue de tous les supports (régie son, ordinateur, smartphone, téléviseur), grâce à un assemblage de transducteurs, de polymères vibro-tactiles et d'électronique haut de gamme.
} 
Sourds et malentendants comme publics de la musique.

Le statut ambigu des technologies numériques dans une démarche d'accessibilité

individuelles se réalisent et qui rappelle l'espace que forment les sourds lorsqu'ils communiquent en groupe ainsi que le corps des danseurs qui " est au sens premier le médium d'une émotion et d'une énergie » (Caune, 2017, p. 212) contribuent à un dispositif scénique où le fait musical est inextricablement lié à un régime de perception visuelle. Cette articulation produit un effet de sens qui valorise ce régime perceptif, régime que la langue des signes vient enrichir et qui, en retour, concourt à sa légitimité.

C'est donc en laissant déborder la réflexion au-delà du dispositif, afin de l'étudier dans ses relations aux autres éléments de contenu et de contexte, que l'accessibilité par le numérique doit être appréhendée. Cette perspective bouscule au passage la notion de chaîne d'accessibilité utilisée dans les guides pratiques et qui suggère que, malgré le fait que chaque dispositif soit essentiel à l'accessibilité globale, chacun de ces dispositifs est indépendant. Pour autant, malgré sa pertinence, cette articulation entre des musiques, des danses, des pratiques linguistiques et des technologies peut-elle résumer à elle seule ce qu'est l'accessibilité musicale?

\subsection{L'accessibilité musicale comme possibilité}

Au cours des trois soirées passées au festival Hip OPsession, de même qu'au cours de toutes les périodes d'observation, nous avons pu remarquer différentes formes d'engagement produisant une grande variété de rapports à la danse, à la musique, au lieu, aux dispositifs et aux autres festivaliers. Ainsi, certains sourds restaient près du bar pour discuter entre amis sans s'approcher de la scène et sans manifester d'intérêt pour l'interprétation français/LSF diffusée sur les écrans. A contrario, nous avons également rencontré deux spectateurs sourds qui avaient réservé leurs places au premier rang afin d'être le plus près, et dans de bonnes conditions visuelles, des danseurs : «ce que je veux voir c'est les battles. Parfois, je regarde l'interprète mais je ne vais pas passer mon temps à regarder tour à tour la scène et l'écran. " (Mehdi, 24 ans, sourd signant)

Nous avons également pu suivre un petit groupe de trois sourds qui, après une longue discussion, ont testé les sacs à dos vibrants 


\author{
Mélanie HÉNAULT-TESSIER, \\ Thibault CHRISTOPHE et Nathalie NEGREL
}

pendant près d'une demi-heure, puis, en les conservant sur le dos, se sont approchés de la scène pour observer les danseurs et commenter leur performance (ainsi que leur tenue vestimentaire) et ensuite se diriger vers le bar et danser un peu.

Cette description succincte dessine, comme le remarquait déjà Sophie Maisonneuve (2009), différentes figures d'acteurs: le festivalier qui fréquente le festival ; l'auditeur/spectateur qui écoute la musique ou qui regarde le spectacle ; l'amateur qui est engagé dans son amour pour ce qu'il voit ou entend. L'engagement étant par ailleurs un processus dynamique, chaque personne peut tour à tour devenir l'une de ces figures. L'attention est, elle aussi, variable: l'activité d'écoute peut par exemple être active ou passive. La fluidité et la diversité de ces engagements rappellent donc que l'expérience musicale est avant tout une possibilité. En d'autres mots, l'expérience musicale des personnes sourdes et malentendantes est un engagement concret et individuel choisi et aussi un droit - qui repose cependant sur "les garanties collectives de sa réalisation " (Zaffran, 2015, p. 18); ce sont ces garanties que désigne la notion d'accessibilité. Alors que la médiation culturelle est davantage perçue comme un échange et une confrontation entre les subjectivités afin de construire les modalités du "Vivre-ensemble » dans une perspective intégratrice (Caune, 2017), l'accessibilité culturelle désigne un processus d'adaptation et de transformation du collectif permettant aux individualités de s'exprimer et de se réaliser.

\title{
2. "À quoi sert le sac à dos ? ": la mise à l'épreuve des dispositifs numériques d'accessibilité
}

En plus du recours à des technologies ordinaires afin de soutenir l'expression de l'altérité musicale, le numérique est également mobilisé dans des dispositifs hybrides d'amplification sensorielle: sac à dos vibrants, caissons vibrants, colonne lumineuse et vibrante. Ces dispositifs contribuent à déplier le fait sonore, mais certains sourds les jugent inutiles puisque "les vibrations sont déjà dans l'air, elles sont partout». D'autres mentionnent que "sur l'estrade, c'était mieux » en référence aux sensations ressenties sur l'estrade en bois. Ces remarques 
Sourds et malentendants comme publics de la musique. Le statut ambigu des technologies numériques dans une démarche d'accessibilité

montrent que, comme l'expérience musicale, l'accessibilité musicale est une mise à l'épreuve des relations entre des producteurs, des auditeurs, des musiques et des dispositifs. Elles interrogent également le rôle et le statut de ces dispositifs spécifiques dans le processus de mise en accessibilité d'un festival musical.

L'une des premières réponses pouvant être apportées émane des trois chargés de projets que nous avons rencontrés au cours de cette recherche. Ils notent que la présence de technologies au service de l'accessibilité dans les festivals et dans les concerts facilite l'obtention de subventions. L'un d'entre eux a résumé leur rôle et leur statut en quelques mots au cours d'une journée d'étude consacrée aux expériences musicales des sourds et malentendants $^{16}$ : «les technologies, c'est sexy! " Ce constat attire d'abord l'attention sur une activité centrale - et problématique - de l'accessibilité culturelle, soit la recherche de financement (Duranton et Gonthier-Maurin, 2017). II révèle ensuite un certain consensus sur la pertinence de ces technologies, du moins chez les financeurs, qui fait écho au " pro-innovation bias" que remarquait déjà Everett $M$. Rogers en 1962, comme le rappellent Benoît Godin et Dominique Vinck (2017). Les technologies sont en effet aujourd'hui associées au progrès et semblent promettre "de nous libérer des "exigences du corps et de la matérialité" " (Moser, 2005, p. 144). Le fait que le consensus se fasse autour de dispositifs technologiques d'amplification sensorielle plutôt que sur l'inscription durable et diversifiée de la LSF au sein du festival suggère que les logiques de financement promeuvent davantage les technologies comme outils permettant de compenser « un appareil corporel hors d'état de fonctionner convenablement » (Moser, 2005, p. 134) ou, mieux encore, augmentant l'individu ${ }^{17}$ que les pratiques linguistiques ou culturelles spécifiques.

\footnotetext{
${ }^{16}$ La journée M.U.S.E \#4 « La musique au-delà de l'oreille. Sourd.e.s et expérience musicale » s'est tenue à Toulouse le 15 novembre 2017.

17 Les sacs à dos vibrants ont initialement été conçus pour les professionnels de la musique afin de répondre aux problèmes liées au home studio.
} 


\section{Mélanie HÉNAULT-TESSIER, Thibault CHRISTOPHE et Nathalie NEGREL}

Cette utilisation des technologies les ramène à un statut d'aide technique qui réactualise une approche médicale dénoncée par certaines personnes sourdes 18 et prolonge la logique d'appareillage dans laquelle s'inscrivent les prothèses auditives, la boucle magnétique ou encore l'implant cochléaire. Elle renouvelle également la perspective intégratrice portée sur la culture utilisée comme moyen de mise en commun. Néanmoins, puisqu'il « ne se passe rien tant que la technologie n'est pas dotée de sens, inscrite dans des pratiques, rendue utile et utilisable ainsi que valorisée et légitimée par les acteurs " (Vinck, 2008, p. 91), une deuxième réponse peut également être apportée à partir des pratiques situées. L'analyse de leur mise à l'épreuve individuelle et collective lors des soirées battles et des concerts permet ainsi de mieux cerner comment les utilisateurs sourds et malentendants construisent le sens de ces dispositifs. Plus avant, et puisque « la relation fait l'objet et non l'inverse "(Hennion, 2013, paragr. 2), c'est aussi le sens même de ces dispositifs que l'analyse de cette relation permet d'aborder.

\subsection{Dispositifs et dispositions : une rencontre singulière}

Dans la grande salle du Lieu Unique de Nantes qui accueille les battles, un petit stand a été aménagé, entre le stand des produits dérivés du festival et le stand d'accessoires vestimentaires (très couru pour ses casquettes), afin de permettre la distribution des sacs à dos vibrants. La personne responsable de les distribuer et d'en expliquer le fonctionnement ne parle pas la langue des signes, ce qui a été relevé par de nombreuses personnes sourdes qui lui reprochent de ne pas être en mesure de leur expliquer dans leur langue à quoi sert le sac à dos.

Ainsi, ce dispositif d'amplification des vibrations sonores ne fait pas naturellement sens pour les sourds et malentendants. Contrairement aux idées reçues, les qualités sensibles du corps

\footnotetext{
${ }^{18}$ L'appareillage et la chirurgie cochléaire sont perçus par certains sourds comme un déni de leur langue et de leurs compétences sociolinguistiques (Dalle-Nazébi et Lachance, 2008). Ils contestent une réactualisation du modèle médical de prise en compte du handicap envisagée comme une action de réparation sur un individu que l'on équipe d'une " béquille » afin de compenser les pertes auditives engendrées par un organe défectueux.
} 
Sourds et malentendants comme publics de la musique.

Le statut ambigu des technologies numériques dans une démarche d'accessibilité

des sourds ne garantissent pas une expérience incarnée de la musique. Les entretiens réalisés au cours de cette étude confirment cette observation. Ils ont montré, comme le révèle l'extrait suivant, que loin d'être une évidence, la sensibilité aux vibrations varie d'une personne à l'autre :

Je sais qu'il y a des choses qui sont des musiques douces, un peu comme des textures qui sont différentes. Et... je remarque bien que selon le style de musique, il y a des formes des vibrations différentes, en tout cas des ressentis différents. Mais c'est des choses que j'ai jamais appris à séparer, à discriminer. (Caroline, 42 ans, sourde signante)

Cette citation démontre que ressentir les vibrations est une chose, mais qu'être capable de les utiliser comme instrument du goût et du plaisir en est une autre. Comme cela a été dit dans les études sur les amateurs de musique, pour être agi, pour que les choses prennent, il est nécessaire de se les faire aimer (Hennion, Maisonneuve et Gomart, 2000 ; Teil 2003). Le rôle attribué au sac à dos varie donc selon les capacités sensibles individuelles. À l'inverse de ceux qui remettent en question sa pertinence, certains l'apprécient justement parce qu'il permet de compenser non pas une déficience physique, mais un investissement préalable qui fait défaut. II ouvre de la sorte un champ de possibles, il fait faire des choses, c'est du moins ce que nous dit l'un des festivaliers quand il affirme qu'» avec le sac à dos, je pourrais peu à peu apprendre à danser $"$.

L'expérience incarnée de la musique naît donc d'un engagement qui ne doit pas seulement être compris en termes d'attention, mais aussi en termes d'entraînement, dans lequel interviennent souvent des technologies numériques d'usage courant ${ }^{19}$, permettant de développer des savoir-faire spécifiques sur lesquels les personnes peuvent s'appuyer pour faire émerger les goûts et les plaisirs

\footnotetext{
${ }^{19}$ L'écoute de la musique à partir d'un ordinateur connecté à Internet et équipé d'enceintes acoustiques puissantes est une stratégie déployée par certaines personnes sourdes et malentendantes pour travailler leur sensibilité aux vibrations. Ce dispositif leur permet d'articuler les éléments sonores des musiques - diffusés à plein volume - à leurs éléments visuels comme les vidéoclips et les sous-titres. En écoutant (avec les yeux, les oreilles et le corps) différents morceaux et en comparant leur rythmicité, elles incorporent leur musicalité et affinent leur perception.
} 
Mélanie HÉNAULT-TESSIER, Thibault CHRISTOPHE et Nathalie NEGREL

musicaux. Cet investissement de soi dans la musique montre que l'accessibilité musicale n'est pas un mouvement unidirectionnel révélant, grâce à la médiation technique, une œuvre à un public, mais un mouvement à double sens naissant de la rencontre d'un cadre proposé et d'un engagement de soi lors de laquelle "se joue tout l'enjeu de la "prise", comme art de prendre et art d'être pris » (Maisonneuve, 2009, p. 91).

\subsection{Musique et technologies : des expériences partagées}

La médiatrice culturelle sourde signante présente au cours des soirées joue un rôle important dans l'appropriation des sacs à dos vibrants, puisqu'elle met les festivaliers en contact avec le dispositif à travers d'une relation en LSF. De plus, ses commentaires s'accompagnent d'invitations à poser la main sur le sac pour ressentir les vibrations ou à essayer celui qu'elle porte sur le dos. Il circule ainsi de main en main, de dos à dos, en plus d'être retourné pour être testé sur le thorax. En petits groupes de trois ou quatre, chacun témoigne de son ressenti, encourage certaines utilisations et en discrédite d'autres : certains évoquent une sensation désagréable provoquée par les vibrations circulant sur la colonne vertébrale, quelques-uns mentionnent que, " pour les sourds, c'est gênant d'être sollicité dans le dos ${ }^{20}$ ". Inversement, certains s'émerveillent et affirment que les vibrations " réveillent leur corps " ou encore qu'il " permet de relier le corps au cerveau ». Comme c'est le cas pour toute performance sonore, l'expérience vibratoire s'élabore en partie sur une interprétation collective qui participe de la construction du sens de ce dispositif.

Les dispositifs sont également comparés les uns aux autres. Les vibrations qu'émettent la colonne lumineuse et le caisson vibrant sont dans l'ensemble jugées plus grossières que celles retransmises par le sac à dos. Le commentaire le plus récurrent est toutefois que ces deux dispositifs sont positionnés trop loin de la scène et qu'ils immobilisent et isolent leur utilisateur. En dehors

\footnotetext{
${ }^{20}$ Chez les sourds signants, les rapports à l'environnement physique et humain sont de nature visuelle. Ce lien visuel engage un ensemble de manières de faire et de repères partagés qui s'apprennent, circulent et s'actualisent dans les interactions entre sourds. Les éléments qui échappent à ce lien sont souvent péjorativement connotés puisqu'ils renvoient à des éléments qui ne peuvent être contrôlés ou qui sont imprévisibles.
} 
Sourds et malentendants comme publics de la musique.

Le statut ambigu des technologies numériques dans une démarche d'accessibilité

de quelques brefs essais, ils sont conséquemment restés inutilisés au cours des trois soirées auxquelles nous avons participé. Ce non-usage actualise la musique comme une expérience partagée. Au-delà de la dimension collective de l'expérience musicale, ce que mettent au jour ces utilisations et ces processus d'appropriation est que l'accessibilité culturelle doit être comprise non pas uniquement en termes de partage de contenu, même si un festival, comme une visite au musée, sont des moments où circulent des repères, des codes et des valeurs, mais aussi en termes de participation, puisqu'il est possible d'envisager qu'il « n'y a rien à transmettre, à faire connaître, à partager, que le partage lui-même » (Hennion, cité dans Thonon, 2013, p. 30).

\subsection{Des interrelations qui soutiennent les capacités individuelles}

Avec les dispositifs numériques, tout comme avec la musique, rien n'est donc joué d'avance. Les savoir-faire individuels, le collectif, l'inscription spatiale et les autres dispositifs sont autant d'éléments qui font sens en situation. Leur rôle et leur statut au sein d'une démarche d'accessibilité sont donc fondamentalement relationnels.

Selon ce que nous disent unanimement les sourds et malentendants, rencontrés au cours du festival Hip OPsession et lors des entretiens individuels, le "plaisir » que procurent les vibrations naît surtout de leur articulation à ce qui est vu et à ce qui peut être entendu ${ }^{21}$. Ressenties seules, les vibrations ont une capacité d'émouvoir qui reste limitée. Associées à des séquences de langue des signes, à l'enchaînement complexe de quelques figures de break dance, au mouvement de la foule qui répond à la musique comme un seul grand corps ou encore à des nuances sonores, elles font naître des émotions tout comme elles en deviennent le support. Dans un environnement aménagé pour permettre un rapport visuel et linguistique à la musique, le sac à

\footnotetext{
${ }^{21}$ La relation au fait sonore donne lieu, chez certains sourds et malentendants, à un jeu de prothèses qui consiste à mobiliser son appareillage lorsqu'ils souhaitent créer une relation auditive avec l'environnement et à les enlever à certaines occasions afin, par exemple, d'explorer toutes les subtilités des vibrations sonores.
} 


\author{
Mélanie HÉNAULT-TESSIER, \\ Thibault CHRISTOPHE et Nathalie NEGREL
}

dos vibrant, qui permet de s'approcher de la scène ou de se positionner stratégiquement face à un écran sur lequel est diffusée l'interprétation en LSF sans avoir à se préoccuper de son positionnement par rapport aux enceintes acoustiques, soutient ce travail d'articulation.

L'accessibilité musicale ne peut se résumer en un objet, un caisson ou un gilet vibrant. Elle est au contraire un espace dans lequel des objets, des technologies, des humains, des standards, des modes et des modalités de communications sont interreliés à travers des processus d'adaptation et de transformation des pratiques et de l'environnement matériel. Les relations que compose cet espace soutiennent alors les personnes, leurs qualités et leurs capacités (Winance, à paraître).

\title{
Conclusion
}

L'analyse située de l'activité d'écoute musicale des personnes sourdes et malentendantes lors d'un festival de musique hip-hop a permis d'observer une traduction en termes pratiques de ce qui est politiquement et institutionnellement qualifié d'accessibilité culturelle. En s'intéressant aux rapports - au fait sonore, aux objets, aux autres ainsi qu'à l'espace - qui actualisent l'écoute musicale, les expériences musicales des personnes sourdes et malentendantes sont apparues comme l'expression d'une altérité sensorielle. Toutefois, pour les sourds comme entendants, l'écoute musicale demeure une rencontre incertaine entre des dispositifs et des dispositions.

L'accessibilité musicale ne peut donc pas se résumer à une recomposition sociotechnique de la musique, comme elle ne peut non plus être réduite à la possibilité d'aller dans un lieu, ou à une série d'actions donnant au public " un véritable accès à l'œuvre, pour qu'il ait les moyens de la comprendre, de la vivre » (North, 2016 , p. 6). L'accessibilité musicale est en effet tout cela à la fois, en plus d'être aussi une possibilité d'exprimer une altérité sensorielle, un investissement individuel, une occasion d'observer, d'expérimenter, de se saisir de pratiques partagées, ainsi qu'une expérience collective qui réaffirme l'appartenance à ce ou ces 
Sourds et malentendants comme publics de la musique.

Le statut ambigu des technologies numériques dans une démarche d'accessibilité

mêmes collectifs. Comme le formule Maxime Cervulle (2013) à propos de la notion de diversité, la notion d'accessibilité "fait partie de ces mots qui semblent autant occulter qu'ils ne révèlent " (p. 104). Si elle est " un moyen de placer l'usager au cœur du projet » (Sophys-Véret, 2015, p. 106) en invitant à un travail de repérage de ce qui crée des incapacités et freine la participation, elle échoue cependant à dire qu'elle est, comme la musique, une " rencontre entre un dispositif et des "arts de faire" " (Maisonneuve, 2009, p. 113).

En tant qu'elle s'actualise à travers des arts de faire, l'accessibilité musicale ne peut conséquemment être confondue avec l'égalité d'expérience ou encore la mise en commun des sensibilités que tendent à promouvoir les technologies telles que la boucle magnétique et, dans une certaine mesure, le sac à dos vibrant. L'égalité d'expérience est en fait une possibilité parmi d'autres puisque, comme le montrent les pratiques d'écoute des sourds et malentendants, l'accessibilité se joue aussi au-delà de l'oreille et des questions d'appareillage, d'implant et de rééducation auditive. Elle consiste donc à prendre acte du caractère multiple de l'expérience, à le soutenir en multipliant les prises afin de créer des espaces où chacun, selon ses envies et ses compétences, est libre de se saisir des données sensibles, des informations textuelles ou linguistiques qui lui sont proposées et de les agencer comme il le souhaite. Cette façon d'appréhender l'accessibilité est éloignée de son acception initiale. Celle-ci induit en effet un accès à un espace finalisé alors que son acception élargie contient une part d'imprévisible.

Afin de distinguer l'accès physique d'un processus d'enrichissement d'une production culturelle visant à encourager son appropriation par le plus grand nombre, nous différencions l'accessibilité de l'appropriabilité. Cette distinction sémantique permet par ailleurs de mieux penser la question du numérique jusqu'alors diluée par la nature polysémique et polymorphe de l'accessibilité. Elle permet ainsi d'interroger les technologies numériques en termes clairs sur leur capacité à développer l'appropriabilité d'une œuvre, d'une production culturelle. 


\section{Mélanie HÉNAULT-TESSIER, Thibault CHRISTOPHE et Nathalie NEGREL}

En matière d'expérience musicale, les technologies numériques soulèvent des enjeux spécifiques tout en étant à même de porter, voire d'amplifier des tendances contradictoires (Boullier, 2016). Celles-ci peuvent ainsi contribuer à l'appropriabilité musicale comme à la réactualisation d'une vision normative de l'activité d'écoute. Agencées à un environnement physique, sensoriel, communicationnel réaménagé, elles peuvent en effet être l'expression matérielle d'une autre manière d'être au monde qu'elles contribuent à légitimer. Cependant, leur nature paradoxale leur permet également d'être appréhendées par certains acteurs comme des prothèses qui réaffirment et matérialisent la surdité comme une expérience problématique caractérisée par l'absence de sons ${ }^{22}$ (Harold, 2013). Les technologies contribuent donc à définir ce que sont l'accessibilité et l'appropriabilité et ce qu'elles devraient être, et, par extension, elles définissent leurs utilisateurs (Moser et Law, 2003). À la suite d'Yves Jeanneret (2017), nous pouvons ainsi évoquer la "responsabilité médiatique » (p.662) des dispositifs numériques puisqu'ils concourent à la construction et à la diffusion de perspectives sur les sourds comme "êtres porteurs d'une différence et non comme des déficients" (Benvenuto, 2004, p. 74) ou, au contraire, promeuvent l'inverse.

Dans les deux cas, la question du rôle et du statut des technologies se pose en relation à la problématique de la participation des personnes sourdes et malentendantes à une pratique culturelle (la musique) partagée par la majorité entendante. Les technologies numériques détournent donc l'attention d'autres pratiques spécifiquement visuelles, telles que le théâtre ou la poésie en LSF, qui pourraient parallèlement être davantage soutenues et valorisées.

\section{Références}

Albrecht, G. L., Ravaud, J.-F. et Stiker, H.-J. (2001). L'émergence des disability studies : état des lieux et perspectives. Sciences sociales et santé, 19(4), 43-73.

\footnotetext{
${ }^{22}$ Gill Harold (2013) remarque en effet que la surdité n'a de cesse d'être appréhendée comme un problème : " D/deafness is a problem, understood only in negative terms as a non-hearing experience characterised by the absence of sound » (p. 860).
} 
Sourds et malentendants comme publics de la musique. Le statut ambigu des technologies numériques dans une démarche d'accessibilité

Benvenuto, A. (2004). De quoi parlons-nous quand nous parlons de " sourds » ?. Le Télémaque, 1(25), 73-86.

Bessy, C. et Chateauraynaud, C. (1993). Les ressorts de l'expertise. Épreuve d'authenticité et engagement des corps. Raisons pratiques, (4), 141-164.

Boidy, M., Meursault p. et Pailler, F. (2015). (Ré)visions du sonore. Politiques sonores, (11), 6-11.

Boullier, D. (2016). Sociologie du numérique. Paris, France : Armand Colin.

Brétéché, S. (2015). L'incarnation musicale. L'expérience musicale sourde (Thèse de doctorat), Aix-Marseille Université.

Caune, J. (2017). La médiation culturelle. Expérience esthétique et construction du Vivre-ensemble. Grenoble, France : Presses universitaires de Grenoble.

Cervulle, M. (2013). Dans le blanc des yeux. Diversité, Racisme et Médias. Paris, France : Éditions Amsterdam.

Cheyronnaud. J. (2009). Préface. Pour une ethnographie de la "forme musique ». Dans A. Pecqueux, et O. Roueff (dir.), Écologie sociale de l'oreille. Enquêtes sur l'expérience musicale (p. 7-12). Paris : EHESS.

Comité des droits économiques, sociaux et culturels. (2009). Observation générale $n^{\circ} 21$. Droit de chacun de participer à la vie culturelle. Quarante-troisième session. Repéré à https://droitsculturels.org/ressources/wpcontent/uploads/sites/2/2012/07/OBSERVATION_GENERALE_ 21-droits-culturels.pdf

Dalle-Nazébi, S. et Lachance, N. (2008). Sourd et médecine : impact des représentations sur les conditions d'accès aux soins. Regards croisés France-Québec. Interrogations, (6), 78-94.

Darras, B. et Valente, D. (2013). La communication autrement. Médiation et Information (MEl), (36), 7-9.

Davallon, J. (2003). La médiation : la communication en procès ?. Médiation et Information (MEI), (19), 37-59. 
Mélanie HÉNAULT-TESSIER, Thibault CHRISTOPHE et Nathalie NEGREL

Dubois, V. et Ebersold, S. (2013). Handicap, pratiques culturelles et participation sociale : rapport de recherche. CNSA, DREESMiRe.

Duranton, N. et Gonthier-Maurin, B. (2017). Culture et handicap : une exigence démocratique (Rapport d'information fait au nom de la commission de la culture, de l'éducation et de la communication par le groupe de travail "Culture et handicap »). Sénat, France : Les Rapports du Sénat.

Fertier, A. (2013). Pour le respect des droits culturels. Hommes et libertés, (163), 37-39.

Godin, B. et Vinck, D. (2017). Critical Studies of Innovation. Alternative Approaches to the Pro-Innovation Bias. Cheltenhank, Angleterre/Northhampton, MA : Edward Elgar Publishing.

Harold, G. (2013). Reconsidering sound and the city: Asserting the right to the Deaf-friendly city. Environment and Planning $D$ : Society and Space, 31, 846-862.

Hénault-Tessier, M. et Dalle-Nazébi, S. (2013). TIC et relation administrative. Repenser l'activité, co-construire l'accessibilité. Dans R. Patesson (dir.), Transformation des organisations. Évolution des problématiques et mutations fonctionnelles (p. 261-270). Bruxelles, Belgique : Éditions de l'Université de Bruxelles.

Hennion, A. (1993). La Passion musicale. Une sociologie de la médiation. Paris, France : Métailié.

Hennion, A. (2004). Les usagers de la musique. L'écoute des amateurs. Circuit : musiques contemporaines, 14(18), 16-31.

Hennion, A. (2013). D'une sociologie de la médiation à une pragmatique des attachements. SociologieS. Repéré à https://journals.openedition.org/sociologies/4353

Hennion, A., Maisonneuve, S. et Gomart, E. (2000). Figures de l'amateur. Formes, objets, pratiques de l'amour de la musique aujourd'hui. Paris, France : La Documentation française. 
Sourds et malentendants comme publics de la musique. Le statut ambigu des technologies numériques dans une démarche d'accessibilité

Houriez, S., Houriez, J., Kounakou, K. et Leleu-Merviel, S. (2013). Accessibilité des musées : de la conception pour les enfants sourds au design for all. Médiation et Information (MEI), (36), 25-37.

Jeanneret, Y. (2014). Critique de la trivialité. Les médiations de la communication, enjeu de pouvoir. Paris, France : Éditions Non Standard.

Kauffmann, I. (2004). Musique et danse hip-hop, des liens étroits à l'épreuve de la professionnalisation. Volume !, 3(2), 73-91.

Larrouy, M. (2007). L'invention de l'accessibilité. Des politiques de transports des personnes handicapées aux politiques d'accessibilité de 1975 à 2005 (Thèse de doctorat). Université Paris I - Panthéon - Sorbonne.

Maisonneuve, S. (2009). L'expérience festivalière. Dispositifs esthétiques et arts de faire advenir le goût. Dans A. Pecqueux et O. Roueff (dir.), Écologie sociale de l'oreille. Enquêtes sur l'expérience musicale (p. 85-115). Paris, France : EHESS.

Maisonneuve, S. (2012). Techno-logies musicales. Communications, 91(2), 77-92.

Moser, I. (2005). De la normalisation aux cyborg studies : comment repenser le handicap. Cahiers du Genre, 1(38), 127162.

Ministère de la Santé et des Solidarités, Ministère délégué à la Sécurité sociale, aux Personnes âgées, aux Personnes handicapées et à la Famille. (2006). Définition de l'accessibilité. Une démarche interministérielle. Repéré à http://www.handipole.org/IMG/pdf/guide_accessibilite_DIPH.pdf

Moser, I., Law, J. (2003). "Making Voices”: New Media Technologies, Disabilities, and Articulation. Dans G. Liestøl, A. Morrison et T. Rasmussen (dir.), Digital Media Revisited: Theoretical and Conceptual Innovation in Digital Domain (p. 491-520). Cambridge, MA : MIT Press.

Nations Unis. (1984). Programme d'action mondial concernant les personnes handicapées. Assemblée générale des Nations 
Mélanie HÉNAULT-TESSIER, Thibault CHRISTOPHE et Nathalie NEGREL

Unies, $37^{\text {ème }}$ session, résolution $37 / 52,3$ décembre. Repéré à http://www.un.org/french/esa/social/disabled/PDF/Programme_a ction mondial.pdf

North, X. (2016). Pour une politique culturelle de l'accès. L'Observatoire, 1(47), 3-7.

Nowak, R. (2013). Consommer la musique à l'ère du numérique : vers une analyse des environnements sonores. Volume !, 10 (1). doi: $10.4000 /$ teth.1030

Pecqueux, A. et Roueff, O. (2009). Introduction. Dans A. Pecqueux et O. Roueff (dir.), Écologie sociale de l'oreille. Enquêtes sur l'expérience musicale (p. 13-26). Paris, France : EHESS.

Plaisance, E. (2015). Culture et Handicap. Les enjeux de l'accessibilité à la culture. Dans J. Zaffran (dir.), Accessibilité et handicap (p. 73-89). Grenoble, France : Presses universitaires de Grenoble.

Sophys-Véret, S. (2015). L'accessibilité à la culture comme vecteur d'un renouvellement des pratiques interprofessionnelles. Dans J. Zaffran (dir.), Accessibilité et handicap (p. 91-106). Grenoble, France : Presses universitaires de Grenoble.

Teil, G. (2003), Aimer le vin : pratiques de la perception. Toulouse, France : Éditions Octarès.

Thonon, M. (2003). Entretien avec Jean Caune, Bernard Darras et Antoine Hennion. Médiation et Information (MEI), (19), 11-34.

Vinck, D. (2008). Conceptualiser l'équipement de l'organisation. Dans D. Vinck et B. Penz (dir.). L'équipement de l'organisation industrielle : les ERP à l'usage (p. 87-100). Paris, France :

Lavoisier.

Winance, M. (à paraître). Introduction. Dans C. Popescu et N. Rapegno (dir.). Géographies du handicap. Paris, France : Collection MSH Paris Nord et OpenEdition.

Zaffran, J. (2015). L'accessibilité est-elle soluble dans l'accès?. Dans J. Zaffran (dir.). Accessibilité et handicap (p. 15-24). Grenoble, France : Presses universitaires de Grenoble. 